\title{
A INFLUÊNCIA DOS ASPECTOS ECONÔMICOS E SOCIAIS NA EVOLUÇÃO DA GESTAÇÃO COM FOCO NA RUPTURA PREMATURA DE MEMBRANAS ${ }^{1}$
}

\author{
Carla Kellen Lima Sousa ${ }^{2}$ \\ Walicy Cosse Silva ${ }^{3}$ \\ Debora Cosse Silva ${ }^{4}$ \\ Domenicke Sarron Lima Sousa ${ }^{5}$ \\ Karla Joelma Bezerra Cunha ${ }^{6}$
}

\section{RESUMO}

A ruptura prematura de membranas amnióticas que recobrem e guardam o feto durante a gestação tem etiologias diferentes, onde destaca-se a condição socioeconômica como um possível preditor para essa ocorrência. Assim, o estudo tem como objetivo analisar o impacto dos fatores sociais e econômicos para a ocorrência da ruptura prematura de membranas. Foi realizado um estudo descritivo com abordagem quantitativa, realizado com mulheres com diagnóstico de ruptura prematura de membrana em uma maternidade de Teresina, PI. Foram entrevistadas 90 mulheres que assinaram o TCLE. Os resultados das variáveis socioeconômicas mostraram que 62 mulheres $(68,9 \%)$ tinham idade entre 20 e 35 anos, 61 mulheres eram pardas $(67,8 \%)$, enquanto 69 delas $(76,7 \%)$ não tinham empregos e $63(70 \%)$ não possuíam nenhum tipo de renda. 0 nível de escolaridade mais alto foi o ensino fundamental para 41 mulheres $(45,6 \%)$ e ensino médio para outras 41 participantes $(45,6 \%)$. $O$ interior do estado do Piauí foi a procedência de 46 mulheres $(51,1 \%)$. Conclui-se com o estudo que a ruptura prematura de membranas ovulares é uma ocorrência frequente e que está associada a maior risco de complicações materno-fetais. As evidências encontradas sugerem uma relação entre a condição socioeconômica de mulheres gestantes e maior risco de ruptura prematura de membrana.

Palavras-Chave: Ruptura Prematura de Membranas Fetais. Gravidez. Parto.

\section{INTRODUÇÃO}

\footnotetext{
1 Trabalho apresentado no Congresso Brasileiro Ciência e Sociedade (CBCS 2019), promovido pelo Centro Universitário Santo Agostinho, de 03 a 05 de outubro de 2019, em Teresina-PI.

2 Pós-graduanda em Docência do Ensino Superior pelo Centro Universitário UNINOVAFAPI. Bacharel em Enfermagem pelo Centro Universitário Santo Agostinho - UNIFSA. E-mail:

carlakellenenfermmagem@gmail.com

3 Pós-graduando em Docência do Ensino Superior pelo Centro Universitário UNINOVAFAPI. Bacharel em Enfermagem pelo Centro Universitário Santo Agostinho - UNIFSA. E-mail: cosse.wallyce174.wc@gmail.com 4 Discente do Curso de Farmácia pelo Centro Universitário Santo Agostinho - UNIFSA. E-mail: deboracosse996@gmail.com

${ }^{5}$ Discente do Curso de Ciências Contábeis pelo Centro Universitário Internacional - UNINTER. E-mail: dsarron@yahool.com.br

${ }^{6}$ Professora do Curso Bacharel em Enfermagem pelo Centro Universitário Internacional - UNINTER. E-mail: karlaenfa@yahoo.com.br
} 


\section{CONGEESSOC CIENCIAESOCIEDADE

ANAIS CBCS 2019 | 3 a 5 de outubro de 2019 | Centro Universitário Santo Agostinho - Teresina - P|

A saúde é apontada, de acordo com o artigo 196 da Constituição Federal de 1988 (BRASIL, 1988) como um direito para todos os cidadãos, sendo dever do Estado garanti-la a população, e o deve faze-la por meio de políticas sociais e econômicas que diminuam o risco de doença e de outros agravos, com acesso universal e igualitário a todos, nas áreas de promoção, proteção e recuperação.

Contudo, o Brasil é um país que carrega ao longo de sua história intensas formas de desigualdades na educação, saúde, segurança, e demais áreas. Para Albuquerque et al. (2017) as políticas sociais e econômicas, que abordam temas como renda e escolaridade, estão associadas a essa desigualdade. Desigualdade que persiste na atual conjuntura brasileira apesar de iniciativas adotadas ao longo dos anos como as políticas de transferência de renda (por exemplo, o programa bolsa família), aumento do emprego formal, valorização do salário mínimo, direcionamento de recursos para a educação, entre outras ações.

Nesse contexto, as políticas públicas apresentam-se como respostas do Estado aos problemas da sociedade. O Sistema Único de Saúde (SUS), de acordo com Ferraz e Kraiczyk (2010), emerge como uma importante política de saúde pública, que incorpora em sua proposta os princípios da universalidade e equidade no acesso aos serviços e ações de saúde, bem como a integralidade da atenção, tendo como diretrizes a descentralização, regionalização e hierarquização do cuidado e de participação da comunidade. Contudo, Albuquerque et al. (2017) aponta como problema o fato de que, por vezes, esse sistema expressa e reproduz em seu funcionamento as desigualdades regionais no Brasil.

É nesse sistema de saúde que estão presentes as políticas voltadas para a saúde da mulher, conquistadas por meio de intensas lutas sociais. É nessa conjuntura que aparece o pré-natal, que consiste na realização de um adequado acompanhamento na unidade básica de saúde (UBS) com captação precoce da gestante, o acolhimento às intercorrências na gestação, a avaliação e classificação de risco e vulnerabilidade, caso a gestante se enquadre na classificação de baixo risco o pré-natal será acompanhado cuidadosamente pelo profissional enfermeiro na UBS (BRASIL, 2012; BRASIL, 2001). 


\section{conghESSO CIENCIAESOCIEDADE \\ Inovação, Diversiliade e Sustentahililitade}

ANAIS CBCS 2019 | 3 a 5 de outubro de 2019 | Centro Universitário Santo Agostinho - Teresina - P |

Segundo Mano et al. (2011) e Dias-da-Costa et al. (2013) a inadequação do pré-natal pelas gestantes está diretamente relacionada a pobreza, baixa escolaridade das mães, baixo poder aquisitivo das famílias, elevada aglomeração domiciliar, péssimas condições de habitação e saneamento, mostrando que quanto maior o número de filhos na família menor a aderência das mulheres ao pré-natal aumentando assim os riscos durante o período gestacional.

Desses fatores surge o risco para aparecimento de complicações à saúde da mulher em todas as fases da vida, mas que aparecem com maior impacto no período gravídicopuerperal. Nesse cenário, a ruptura prematura de membranas amnióticas, que recobrem e guardam o feto durante a gestação, tem etiologias diferentes que incluem as infecções geniturinárias, deficiência nutricional materna, condição socioeconômica, traumas durante a gestação, distensão uterina devido a gestação gemelar, polidramnia, macrossomia fetal, entre outras causas. Para ACOG (2007) e Cobo et al. (2011) a rotura prematura de membranas em fetos a termo ocorre em $8 \%$ das gestações e em pré-termo de 2 a $4 \%$ e sua relevância torna-se evidente devido às complicações devido à prematuridade, morbidade e mortalidade que isso implica.

A importância de discutir essa temática se dá pelas repercussões que essa ocorrência pode ocasionar à gestante e ao feto, como infecções, parto prematuro e complicações pós-parto. Assim, este estudo analisa o impacto dos fatores sociais e econômicos para a ocorrência da ruptura prematura de membranas para responder ao questionamento: Quais as repercussões da situação socioeconômica para a saúde do binómio mãe/filho e ocorrência da ruptura prematura de membranas?

\section{METODOLOGIA}

Realizou-se uma pesquisa descritiva, de abordagem quantitativa, desenvolvida em uma maternidade pública de referência em Teresina. Participaram da pesquisa mulheres diagnosticadas com rotura prematura de membranas ovulares, internadas na maternidade 


\section{CONGEESSOC CIENCIAESOCIEDADE

ANAIS CBCS 2019 | 3 a 5 de outubro de 2019 | Centro Universitário Santo Agostinho - Teresina - P|

no período da coleta dos dados, e que consentiram em participar com assinatura do Termo de Consentimento Livre e Esclarecido - TCLE. Foram excluídas da amostra as mulheres sem diagnóstico de rotura prematura de membranas ovulares, mulheres com transtorno mental, as que foram internados fora do período da pesquisa, e não aceitarem participar da pesquisa. O quantitativo final da amostra foi de 90 mulheres.

A coleta dos dados foi realizada com entrevista e preenchimento de um formulário semiestruturado com perguntas abertas e fechadas objetivando complementar as informações existentes nos prontuários, respeitando os objetivos da pesquisa. As informações contidas no formulário que foram usadas para esse recorte dizem respeito às variáveis sociodemográficas como: idade, renda familiar, escolaridade, ocupação/profissão, raça, procedência. A análise das variáveis foi realizada no software SPSS (Statistical Package for Social Sciences) versão 19.0. A pesquisa foi realizada em acordo com os princípios apresentados na Resolução no 466 de 2012 do Conselho Nacional de Saúde (BRASIL, 2012).

\section{RESULTADOS E DISCUSSÃO}

A análise das variáveis sociodemográficas das mulheres no período pós-parto por meio de entrevistas realizadas permitiu a elaboração da tabela abaixo contendo os dados estatísticos obtidos.

Tabela 1 - Apresentação das variáveis sociodemográficas de mulheres com rotura precoce de membranas ovulares $(n=90)$. Teresina, 2019.

\begin{tabular}{lcc}
\hline Variáveis & $\mathbf{N}$ & $\%$ \\
\hline Idade & & \\
15 a 19 anos & 20 & 22,2 \\
20 a 35 anos & 62 & 68,9 \\
Mais de 35 anos & 8 & 8,9 \\
Raça & & \\
Branca & 14 & 15,6 \\
Preta & 15 & 16,7 \\
\hline
\end{tabular}




\section{CONGEESSOC CIENCIAESOCIEDADE

ANAIS CBCS 2019 | 3 a 5 de outubro de 2019 | Centro Universitário Santo Agostinho - Teresina - P|

\begin{tabular}{lcc}
\hline Parda & 61 & 67,8 \\
Ocupação & 21 & 23,3 \\
Sim & 69 & 76,7 \\
Não & & \\
Renda & 27 & 30,0 \\
Sim & 63 & 70,0 \\
Não & & \\
Escolaridade & 41 & 45,6 \\
Ensino fundamental & 41 & 45,6 \\
Ensino médio & 8 & 8,9 \\
Ensino superior & & 41,1 \\
Procedência & 37 & 51,1 \\
Capital PI & 46 & 0 \\
Interior PI & 0 & 7,8 \\
Capital MA & 7 & \\
Interior MA & & \\
\hline
\end{tabular}

Fonte: Pesquisa direta.

A Tabela 1 evidencia que 62 mulheres (68,9\%) tinham idade entre 20 e 35 anos, 61 mulheres eram pardas $(67,8 \%)$, enquanto 69 delas $(76,7 \%)$ não tinham empregos e 63 (70\%) não possuíam nenhum tipo de renda. O nível de escolaridade mais alto foi o ensino fundamental para 41 mulheres $(45,6 \%)$ e ensino médio para outras 41 participantes (45,6\%). O interior do estado do Piauí foi a procedência de 46 mulheres (51,1\%).

De acordo com Lee et al. (2018) a ruptura prematura de membranas fetais é definida como a rotura das membranas ovulares de forma espontânea em período anterior às 37 semanas gestacionais e início do parto. Sua incidência geral é de cerca de $3 \%$ de ocorrência em todas as gestações. Muitos fatores podem participar direta ou indiretamente desse evento. Entre os motivos, a literatura apresenta como principais a presença de mecanismos patológicos como infecções intra-amnióticas, hiperdistensão uterina, bem como fatores obstétricos como rotura prematura de membrana anterior, hábitos comportamentais e condição econômica. É uma condição clinicamente importante por aumentar o risco de infecção após a ruptura, risco de parto prematuro, que pode gerar sequelas e aumento de morbimortalidade neonatal. 


\section{cONGERESOC CIENCIAESOCIEDADE \\ Inovação, Diversidaade e Sustentahililitaile}

ANAIS CBCS 2019 | 3 a 5 de outubro de 2019 | Centro Universitário Santo Agostinho - Teresina - P |

A idade das mulheres do estudo foi de 20 a 35 anos, período em que a mulher se encontra em sua idade fértil com maior possibilidade de concepção. Este dado apresentase em conformidade com números de estudo internacional prospectivo de coorte realizado em um hospital na Uganda com mulheres com ruptura prematura de membranas após 28 semanas de gestação no período de novembro de 2015 e maio de 2016, onde a idade média encontrada foi de 25 anos ( $d p \pm 5,3$ ), com período de idade maior entre 25 e 35 anos (KAYIGA et al., 2018).

Em estudo transversal realizado por Hackenhaar et al. (2014) em um município do Rio Grande do Sul com mulheres que apresentaram perda de líquido amniótico antes do início de trabalho de parto, verificou-se que 69,9\% eram de cor de pele branca. Esse dado contrasta com o apresentado na presente pesquisa, onde a população estudada era da raça parda.

A condições econômica pode apresentar forte influência na evolução da gravidez. Neste sentido, as mulheres do estudo não possuíam ocupação e não dispunham de renda mensal própria para sustento. Os resultados divergem dos encontrados em estudo retrospectivo que comparou a ocorrência de parto pré-termo em mulheres com e sem rotura precoce de membranas, onde verificou-se que na população com a rotura $72,3 \%$ possuíam emprego e renda (BRANDÃO et al., 2015). Já a pesquisa de Mendoza e Herrera (2018) corrobora os dados dessa pesquisa, ao evidenciar em seus resultados um percentual de $82,3 \%$ de mulheres diagnosticadas com ruptura prematura de membrana que possuíam baixo nível socioeconômico.

Associado ao baixo nível econômico, a baixa escolaridade também se apresenta como fator de risco para ocorrência de muitas situações relacionadas à saúde. Neste estudo as mulheres possuíam apenas ensino fundamental ou ensino médio, com baixo índice de mulheres com educação superior. Em estudo transversal retrospectivo realizado em uma maternidade no Rio Branco, Acre, cerca de $29 \%$ das mulheres apresentaram apenas ensino fundamental incompleto (SILVA et al., 2014). 


\section{conghESSO CIENCIAESOCIEDADE \\ Inovação, Diversiliade e Sustentahilitiade}

ANAIS CBCS 2019 | 3 a 5 de outubro de 2019 | Centro Universitário Santo Agostinho - Teresina - P |

Verificou-se que as mulheres eram provenientes principalmente do interior do estado. Esse dado contrasta com o que foi encontrado na pesquisa de Mendoza e Herrera (2018) que apresentou resultados onde $58,8 \%$ das participantes eram procedentes de zona urbana.

Os dados apresentados são importantes pois sugerem a influência da baixa condição socioeconômica no aumento do risco de a mulher sofrer com a ruptura prematura de membranas. O que chama a atenção em razão das muitas conquistas alcançadas pela comunidade feminina, tais como direito à atenção obstétrica, atenção no pré-natal, parto e puerpério, rede cegonha, direito ao Planejamento Familiar, exames de preventivos para diversas doenças, licença-maternidade, entre outros.

Embora a mulher grávida tenha direito a saúde, assegurado constitucionalmente, compreendidos no âmbito do SUS e dos programas de saúde da mulher e criança que foram criados, há de se superar ainda as desigualdades de gênero que ainda persistem, e que contribuem para o aumento desses problemas socioeconômicos que aumentam o risco de complicação para a gestante, como a ruptura de membrana. Para tal é necessário superar a visão da mulher como figura presa ao trabalho doméstico e dependente da figura do homem como provedor (BARBOSA; ALMEIDA JÚNIOR, 2017). E não apenas isso, mas é necessário que o estado se movimente no combata à desigualdade social como um todo, com condições iguais de saúde e educação.

\section{CONSIDERAÇÕES FINAIS}

Conclui-se com o estudo que a ruptura prematura de membranas ovulares é uma ocorrência frequente e que está associada a maior risco de complicações materno-fetais. As evidências encontradas sugerem uma relação entre a condição socioeconômica de mulheres gestantes e maior risco de ruptura prematura de membrana, uma vez que na pesquisa as mulheres eram de raça parda, sem ocupação e renda, com ensino fundamental e médio e procedentes principalmente de regiões do interior. 


\section{cONGERESO CIENCIAESOCIEDADE \\ -}

ANAIS CBCS 2019 | 3 a 5 de outubro de 2019 | Centro Universitário Santo Agostinho - Teresina - P|

Com o risco aumentado por baixas condições socioeconômicas, as mulheres sofrem com maior suscetibilidade para essa ocorrência e risco de complicações materno-fetais como infecções, prematuridade e aumento da morbimortalidade. Assim, tem-se como conclusão que é necessária maior atenção quanto a situação social e econômica das gestantes, ao passo que apenas a criação de políticas e programas de saúde voltadas para o gênero parecem não ser suficientes para erradicar esses problemas. Extinguir a desigualdade de gênero, as desigualdades sociais, combater o baixo nível de escolaridade e oferecer melhores condições dentro das comunidades mais carentes, são ações fundamentais e que, aliadas a políticas de saúde eficientes, reduzirão os problemas de saúde da mulher, a exemplo da ruptura prematura de membranas ovulares, com atendimento integral, humanizado e qualificado à gestante, desde o pré-natal até o puerpério.

\section{REFERÊNCIAS}

ACOG. Committee on Practice Bulletins-Obstetrics. ACOG Practice Bulletin No. 80: premature rupture of membranes. Clinical management guidelines for obstetrician gynecologists. J Obstet Gynecol. v. 109, n. 4, p. 1007-19. 2007. Disponível em: https://doi.org/10.1097/01.AOG.0000263888.69178.1f. Acesso em: 10 set. 2019.

ALBUQUERQUE, M. V. et al. Desigualdades regionais na saúde: mudanças observadas no Brasil de 2000 a 2016. Ciência \& Saúde Coletiva, v. 22, p. 1055-1064, 2017. Disponível em: https://www.scielosp.org/article/csc/2017.v22n4/1055-1064/. Acesso em: 08 set. 2019.

BARBOZA, H. H. G.; JUNIOR, V. A. Al. (Des) Igualdade de gênero: restrições à autonomia da mulher. Pensar-Revista de Ciências Jurídicas, v. 22, n. 1, p. 240-271, 2017. Disponível em: http://periodicos.unifor.br/rpen/article/download/5409/pdf. Acesso em: 09 set. 2019.

BRANDÃO, A. M. S.; et al. Parto pré-termo com e sem rotura prematura de membranas: características maternas, obstétricas e neonatais. Rev Bras Ginecol Obstet, v. 37, n. 9, p. 428-33, 2015. Disponível em: https://www.researchgate.net/profile/Ana_Domingues6/publication/282890796_Parto_ 
ANAIS CBCS 2019 | 3 a 5 de outubro de 2019 | Centro Universitário Santo Agostinho - Teresina - P|

pre-

termo_com_e_sem_rotura_prematura_de_membranas_caracteristicas_maternas_obstet ricas_e_neonatais/links/5630e49808ae3de9381cd1d2.pdf. Acesso em: 10 set. 2019.

BRASIL. Ministério da Saúde. Gestação de Alto Risco: manual técnico, 5 ed., Brasília, DF, 2012. Disponível em:

http://bvsms.saude.gov.br/bvs/publicacoes/manual_tecnico_gestacao_alto_risco.pdf. Acesso em: 08 set. 2019.

BRASIL. Ministério da Saúde. Norma Operacional da Assistência à Saúde / SUS NOAS SUS 01/2001. Disponível

em:<http://bvsms.saude.gov.br/bvs/saudelegis../gm/2001/prt0095_26_01_2001.html>. Acesso em: 10 set. 2019.

BRASIL. Presidência da República. Constituição da República Federativa do Brasil de 1988. Brasília, DF: Presidência da República, 2016. Disponível em:

http://www.planalto.gov.br/ccivil_03/Constituicao/Constituiçao.htm. Acesso em: 10 set. 2019.

COBO, T. et al. Clinical and inflammatory markers in amniotic fluid as predictors of adverse outcomes in preterm premature rupture of membranes. J Obstet Gynecol, v. 205, n. 8, 2011. Disponível em:

http://www.academia.edu/download/41444125/Clinical_and_inflammatory_markers_in _amn20160122-15130-16noikb.pdf. Acesso em: 07 set. 2019.

DIAS-DA-COSTA, J. S et al. Inadequação do pré-natal em áreas pobres no Nordeste. Rev. Bras. Saúde Matern. Infant., Recife, v. 13, n. 2, p. 101-109, abr. / jun., 2013. Disponível em: <http://www.scielo.br/pdf/rbsmi/v13n2/a03v13n2.pdf>. Acesso em: 09 set. 2019.

FERRAZ, D.; KRAICZYK, J. Gênero e Políticas Públicas de Saúde-construindo respostas para o enfrentamento das desigualdades no âmbito do SUS. Revista de Psicologia da UNESP, v. 9, n. 1, p. 70-82, 2017. Disponível em:

http://seer.assis.unesp.br/index.php/psicologia/article/download/428/406. Acesso em: 09 set. 2019.

HACKENHAAR, A. A.; et al. Preterm premature rupture of the fetal membranes: association with sociodemographic factors and maternal genitourinary infections. Jornal de pediatria, v. 90, n. 2, p. 197-202, 2014. Disponível em: https://scholar.google.com.br/scholar?output=instlink\&q=info:TAVkNcffbrAJ:scholar.goo gle.com/\&hl=pt-BR\&as_sdt=0,5\&scillfp=4949699806271082208\&oi=Ile. Acesso em: 09 set. 2019. 
ANAIS CBCS 2019 | 3 a 5 de outubro de 2019 | Centro Universitário Santo Agostinho - Teresina - P|

KAYIGA, H.; et al. Impact of mode of delivery on pregnancy outcomes in women with premature rupture of membranes after 28 weeks of gestation in a low-resource setting: A prospective cohort study. PloS one, v. 13, n. 1, p. e0190388, 2018. Disponível em: https://journals.plos.org/plosone/article/file?id=10.1371/journal.pone.0190388\&type=pr intable. Acesso em: 09 set. 2019.

LEE, Y. J.; et al. Amniotic fluid index, single deepest pocket and transvaginal cervical length: Parameter of predictive delivery latency in preterm premature rupture of membranes. Taiwanese Journal of Obstetrics and Gynecology, v. 57, n. 3, p. 374-378, 2018. Disponível em: https://www.sciencedirect.com/science/article/pii/S1028455918300664/pdfft?md5=529 01df808f4ca3e5b84cc08d175f9f8\&pid=1-s2.0-S1028455918300664-main.pdf. Acesso em: 08 set. 2019.

MANO, P. S. et. al. Iniquidade na assistência à gestação e ao parto em município do semiárido. Rev. Bras. Saúde Matern. Infant. Recife, v. 11, n. 4, p. 381-388 out. / dez, 2011. Disponível em: http://www.scielo.br/pdf/rbsmi/v11n4/v11n4a04. Acesso em: 10 set. 2019.

MENDOZA, S. K. B.; HERRERA, E. G. R. Factores de riesgos de ruptura prematura de membranas. Hospital Provincial General Docente Riobamba. noviembre 2017-febrero 2018. Revista Eugenio Espejo, v. 12, n. 1, p. 53-63, 2018. Disponível em: http://eugenioespejo.unach.edu.ec/index.php/EE/article/view/38. Acesso em: 08 set. 2019.

SILVA, S. M. M.; et al. Morbidade e mortalidade perinatal em gestações que cursaram com amniorrexe prematura em maternidade pública do Norte do Brasil. Rev Bras Ginecol Obstet, v. 36, n. 10, p. 442-448, 2014. Disponível em: http://www.scielo.br/pdf/rbgo/v36n10/0100-7203-rbgo-36-10-00442.pdf. Acesso em: 11 set. 2019. 